\title{
EXOGENOUS APPLICATION OF INORGANIC SALTS DURING GROWTH STAGES INFLUENCES THE VEGETATIVE GROWTH, MARKETABLE YIELD AND QUALITY OF STRAWBERRY (FRAGARIA × ANANASSA) CULTIVAR 'CHANDLER'
}

\author{
SHAHZAD, S. ${ }^{1}-$ AHMAD, S. ${ }^{1 *}-$ RASHID, M. Z. ${ }^{2}-$ SHEHZAD, S. ${ }^{3}-$ SHAFQAT, W. ${ }^{1}-$ HANIF, A. ${ }^{1}$ \\ ${ }^{1}$ Institute of Horticultural Sciences, University of Agriculture, Faisalabad 38000, Pakistan \\ ${ }^{2}$ Horticultural Research Institute, Ayub Agricultural Research Institute, Faisalabad 38850, \\ Pakistan \\ ${ }^{3}$ Department of Plant Pathology, University of Agriculture, Faisalabad 38000, Pakistan \\ * Corresponding author \\ e-mail: saeedsandhu@uaf.edu.pk, sanashahzad247@gmail.com; phone: +92-300-768-8958
}

(Received $9^{\text {th }}$ Dec 2019; accepted 24 ${ }^{\text {th }}$ Mar 2020)

\begin{abstract}
In Pakistan strawberry yield and cultivation area is significantly lower as compared the major strawberry growing countries. Defective agronomic practices cause reduction in yield and quality. This experiment was conducted by using non-chemical techniques including exogenous application of inorganic salts $\mathrm{CaCl}_{2}(3,5$ and $7 \mathrm{mM} / \mathrm{L})$ and $\mathrm{ZnSO}_{4}(50,100$ and $150 \mathrm{mg} / \mathrm{L})$ during growth stages (34 leaves stage and after fruit setting) and their effects on marketable yield and quality of strawberry were documented. The highest number of leaves (18.25), leaf area $\left(47 \mathrm{~cm}^{2}\right)$ and crowns $(7.50)$ were found with $7 \mathrm{mM} / \mathrm{L} \mathrm{CaCl}{ }_{2}$ as compared to control plants. Minimum numbers of days (27.7) for flower anthesis were recorded with $100 \mathrm{mg} / \mathrm{L} \mathrm{ZnSO}$. Maximum marketable yield $\left(348.5 \mathrm{~g} \mathrm{plant}^{-1}\right)$, firmness $\left(0.96 \mathrm{~kg} . \mathrm{cm}^{-2}\right)$,

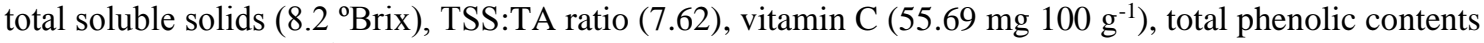
(186.5 mg GAE $100 \mathrm{~g}^{-1}$ ) and higher total antioxidants (75.5\% DPPH) were observed with $7 \mathrm{mM} / \mathrm{L} \mathrm{CaCl}$ application. However, maximum superoxide dismutase activity $\left(30.2 \mathrm{U} \mathrm{mg}^{-1}\right.$ protein) was examined with $100 \mathrm{mg} / \mathrm{L} \mathrm{ZnSO} 4$ while peroxidase activity $\left(1.05 \mathrm{U} \mathrm{mg}^{-1}\right.$ protein) decreased with this concentration. Overall, the response of $7 \mathrm{mM} / \mathrm{L} \mathrm{CaCl}_{2}$ was highly effective for increasing marketable yield and quality. Keywords: calcium chloride, zinc sulfate, total phenolic contents, total antioxidants, marketable yield
\end{abstract}

\section{Introduction}

Strawberry (Fragaria $\times$ ananassa Duch.) a sweet flavored small fruit belonging to the Rosaceae family (Sharma, 2002). Among berry fruits strawberry is most popular and economically important fruit crop (Santos and Chandler, 2009). Nature has blessed Pakistan with different agro climatic conditions which are suitable for production of strawberry. Strawberry plants require low chilling condition and can be planted in wide range of soil types (Asad, 1997). Strawberry is newly emerging small fruit crop in Pakistan; therefore, yield is very lower as compared to other major strawberry producing countries due to improper research techniques and defective field practices (Mabood, 1994). In Pakistan strawberry is cultivated on 179 hectares with 609 tons annual production (GOP, 2015). Strawberries are delicious low-calorie fruits with rich source of vitamins, minerals, anthocyanins and phenolics (Adda Bjarnadottir, 2012).

Rapid growth of strawberry plants (crowns, leaves and runners) can occur within 23 months, which depends on nutrients, light, temperature and water conditions ( $\mathrm{Li}$ et al., 2010). Fast growth habit of strawberry plant demands sufficient supply of macro and micro nutrients synchronized with growth stages of the crop (Phillips, 2004; Medeiros 
et al., 2015). Exogenous application is a supplemental application method to supply nutrients during growth stages when plants cannot uptake adequate nutrients from soil due to complex soil chemistry, leaching of nutrients, low soil temperature, immobile nutrients and low water availability which solubilize the nutrients (Anonymous, 2018; Ghani et al., 2011). In general, through foliar application essential nutrients penetrate in the cuticle or enter through the stomata of leaves more rapidly as compared to soil application (Alshaal and Ramady, 2017). Exogenous application of salts enhances the vegetative growth (plant height, crown diameter, canopy growth, runner growth and leaf area) and also improves the quality and yield of strawberry (Qureshi et al., 2013). In inorganic salts calcium chloride is important which improved the inflexibility of plant cells (Sams, 1999; Maas, 1998). Plants obtain calcium from soil solution through root system via xylem. Deficiency of calcium in root system disturbs the normal life cycle of plants and causes malformation (White, 2000).

Exogenous application of calcium considered as cultural practice for improving fruit calcium contents and it also reduced the decay problem (Bramlage et al., 1985; Elad and Volpin, 1993). Foliar applied calcium chloride before harvest delayed tissue softening, fruit ripening process and reduced grey mould disease in strawberries (Cheour et al., 1990). Zinc sulfate as inorganic salt involved in plant enzymatic reactions and regulate protein and carbohydrate metabolism (Lolaei et al., 2012). Zinc is major component of several enzymes, proteins and important metal element for normal metabolic process of plants. It increased the activity of enzyme called tryptophan which, further enhanced the production of growth hormone called indole acetic acid and acted as growth promoter (Nasiri et al., 2010). Through foliar application zinc played important role for increasing the fruit set, production and quality attributes of fruits and reduced the different physiological disorders in fruit trees (Meena et al., 2014).

In literature, very little information available but not complete answers regarding the effects of foliar application of salts for increasing the marketable yield of strawberry and for improving fruit quality. Foliar application of fungicides is largely used practice in Pakistan which causes the health issues but not focus on non-chemical techniques which reduce the health issues in humans. By keeping in view previous studies, the present study was executed to pursue the following goal: To optimize the best concentration of $\mathrm{CaCl}_{2}$ and $\mathrm{ZnSO}_{4}$ which increase the marketable yield and also improve the quality of strawberry.

\section{Materials and methods}

\section{Experimental site}

Field experiment was conducted at Ayub Agricultural Research Institute (AARI), Jhang Road Faisalabad, Pakistan in the fruit research area during October, 2016 to April, 2017. Raised beds were prepared after one month of soil preparation and their width was $45 \mathrm{~cm}$. Black polyethylene sheet as mulching material was used to cover the beds and for controlling weeds. Plant to plant distance was $25 \mathrm{~cm}$. Healthy and diseasefree bare root transplants of strawberry cv. 'Chandler' were collected from Agricultural Research Institute North Mingora, Swat, Khyber Pakhtoonkhwa. Different foliar applications of salts including $\mathrm{CaCl}_{2}(3,5$ and $7 \mathrm{mM} / \mathrm{L})$ and $\mathrm{ZnSO}_{4}(50,100$ and $150 \mathrm{mg} / \mathrm{L}$ ) were applied on strawberry plants and these were compared with control plants. This experiment was consisted of 7 treatments replicated four times. There were 40 plants in each treatment and total 280 plants used for this experiment. First foliar 
application of salts was applied after 2 weeks of runner transplantation when old leaves were dried and new sprouting occurred at 3-4 leaves stage and second was done at fruit setting stage. Same amount of salt concentration was used in two different growth stages. Each concentration was dissolved separately in one liter of water for 10 plants. For foliar spray $100 \mathrm{ml}$ water was used for single strawberry plant for better absorption and penetration. The surfactant Tween-20 $(0.01 \%)$ was added as wetting agent for foliar application. Foliar application was applied during morning time 6-7 am with handheld sprinkler in a very gentle way.

\section{Vegetative parameters}

Numbers of leaves were counted during growing season. At the end of season three healthy plants from each replication were pull out from beds and then each plant was cut into two halves in such way that trifoliate leaves with petioles separated and below portion crown separated. After cutting of each plant numbers of leaves and crowns were counted. Leaf area of strawberry plants was measured with leaf area meter (LI-COR, 3100C). Numbers of days required for flower anthesis and numbers of runners per plant were also counted after first foliar application (Sangha and Agehara, 2016).

\section{Yield parameters}

Strawberry harvesting was started during end of January and continued till mid-May. Yield was accounted as marketable, unmarketable and small size $\left(\mathrm{g}\right.$ plant $\left.{ }^{-1}\right)$. Strawberries which were $75-80 \%$ fully mature, bright red color, larger size and disease free counted as marketable yield during whole season. Strawberry fruits which were affected due to grey mould disease (Botrytis cinerea), anthracnose fungal disease (Colletotrichum), powdery mildew (Podosphaera aphanis), thrips attack, phyllody (abnormal development of floral parts into leafy structures caused by phytoplasma or virus infections) and due to environmental factors (chilling injury and frost injury) counted as unmarketable yield during season. Strawberries which were $(<10 \mathrm{~g})$ and bullet shaped counted as small size yield during whole season (Sangha and Agehara, 2016).

\section{Fruit quality parameters}

\section{Firmness}

Fruit firmness was measured with digital penetrometer (Humboldt H-1240D) by using $3 \mathrm{~mm}$ diameter probe which measure the penetration force.

\section{Total soluble solids, titratable acidity and TSS:TA ratio}

Total soluble solids ( ${ }^{\circ}$ Brix), TA (\%) and TSS:TA ratio measured with digital TSS/Acid meter (Atago, Japan).

\section{Vitamin C}

For the measurement of vitamin $\mathrm{C}\left(\mathrm{mg} 100 \mathrm{~g}^{-1}\right)$ contents in strawberry extract, first of all strawberry extract was filtered then $(10 \mathrm{ml})$ aliquot was taken in $(100 \mathrm{ml})$ volumetric flask and volume was made up to mark after addition of $(0.4 \%)$ oxalic acid. For titration purpose $(5 \mathrm{ml})$ aliquot was taken in beaker and titration was done with $(2$, 
6-dichlorophenol indophenol) when pink color appeared it was indication of end point. Calculations were done with the method described by Ruck (1969).

\section{Total phenolic contents}

For the determination of TPC of strawberry extract FC method was used. It is called as Folin-Ciocalteu method. Total phenolic contents (mg GAE $100 \mathrm{~g}^{-1}$ ) were determined by using spectrophotometer and read at $765 \mathrm{~nm}$ against the standard curve of Gallic acid $\left(\mathrm{R}^{2}=0.7884\right)$. Calculations were performed according to method as described by Ainsworth and Gillespie (2007).

\section{Total antioxidants}

For TA determinations take supernatant $(50 \mu \mathrm{L})$ and methanolic solution $0.004 \%$ $(5 \mathrm{ml})$ in test tube. Methanolic solution expressed in (2, 2-diphenyl-1 picrylhydrazyl radical) \% DPPH. Samples were tested with the interval of 30 minutes. Changes in absorbance were measured at $517 \mathrm{~nm}$ by microplate reader using spectrophotometer. Calculations were performed by using method described by Brand-Williams et al. (1995).

\section{Activities of anti-oxidative enzymes}

For catalase activity hydrogen peroxide was used to initiate the reaction mixture. Enzyme extract $(100 \mu \mathrm{L})$ was mixed in $\mathrm{H}_{2} \mathrm{O}_{2}(100 \mu \mathrm{L})$ and then activity was observed $240 \mathrm{~nm}$ by microplate reader. Peroxidase activity was measured by using potassium phosphate buffer $(0.1 \mathrm{M})$, hydrogen peroxide $(40 \mathrm{mM})$ and guaiacol $(20 \mathrm{mM})$ with different ratios (8:1:1). Absorbance was noted at $470 \mathrm{~nm}$ by microplate reader using spectrophotometer. Calculations were performed by using method described by Liu et al. (2009). For estimation of superoxide dismutase activity nitro blue tetrazolium was used for 50\% inhibition of photochemical reduction. Absorbance was noted at $560 \mathrm{~nm}$ by using microplate reader. The enzymatic activities were calculated in Unit $\left(\mathrm{U} \mathrm{mg}^{-1}\right.$ protein) by using method described by Jimenez et al. (2003).

\section{Survival}

Diseased, damaged and dead plants were removed from field during entire season. At the end of season remaining plants were counted for plants survival percentage.

$$
\text { Survival } \%=\frac{\text { Number of disease, damage and dead plants }}{\text { Total number of plants }} \times 100
$$

\section{Statistical analysis}

Results were statistically analyzed by using (MINITAB ${ }^{\circledR} 18.0$ and SPSS 21 Software). Randomized Complete Block Design (RCBD) was used for this experiment. To test the overall significance of data ANOVA techniques were employed. To compare the differences among treatment means $(P \leq 0.05)$ Fisher's Least Significant Difference (LSD) test was used (Meier, 2006). 


\section{Results}

\section{Vegetative parameters}

Vegetative growth was significantly increased with exogenous application of salts throughout strawberry season as compared to control treatment (Fig. 1). Maximum number of leaves (18.25) was observed in strawberry plants those were treated with $7 \mathrm{mM} / \mathrm{L} \mathrm{CaCl} 2$ followed by other treatments including $100 \mathrm{mg} / \mathrm{L} \mathrm{ZnSO}$ (15.25), $150 \mathrm{mg} / \mathrm{L} \mathrm{ZnSO}_{4}$ (14.50), $5 \mathrm{mM} / \mathrm{L} \mathrm{CaCl}_{2}$ (13.50), $3 \mathrm{mM} / \mathrm{L} \mathrm{CaCl}_{2}$ (12.0) and $50 \mathrm{mg} / \mathrm{L}$ $\mathrm{ZnSO}_{4}$ (10.75) per plant, respectively (Table 1). Mean values of treatments showed that leaf area $\left(47 \mathrm{~cm}^{2}\right)$ was increased in all treated plants and maximum increment was observed with $7 \mathrm{mM} / \mathrm{L} \mathrm{CaCl}_{2}$ application as compared to non-treated plants. Minimum numbers of days (27.7) for flower anthesis were required when strawberry plants were treated with $100 \mathrm{mg} / \mathrm{L} \mathrm{ZnSO}_{4}$ as compared to control treatment where maximum numbers of days (42.25) were observed for flower anthesis. The response of $\mathrm{ZnSO}_{4}$ foliar application was more for early flower initiation while all $\mathrm{CaCl}_{2}$ treatments were statistically at par with each other. Crown growth was also increased with foliar application of salts and maximum numbers of crowns (7.50) were found with $7 \mathrm{mM} / \mathrm{L}$ $\mathrm{CaCl}_{2}$ as compared to others. The effect of foliar application of salts for increasing runner growth of strawberry plants was found non-significant. Overall, runner growth was increased with foliar application of $\mathrm{CaCl}_{2}$ and $\mathrm{ZnSO}_{4}$ but statistically all treatments means were same (Table 1).

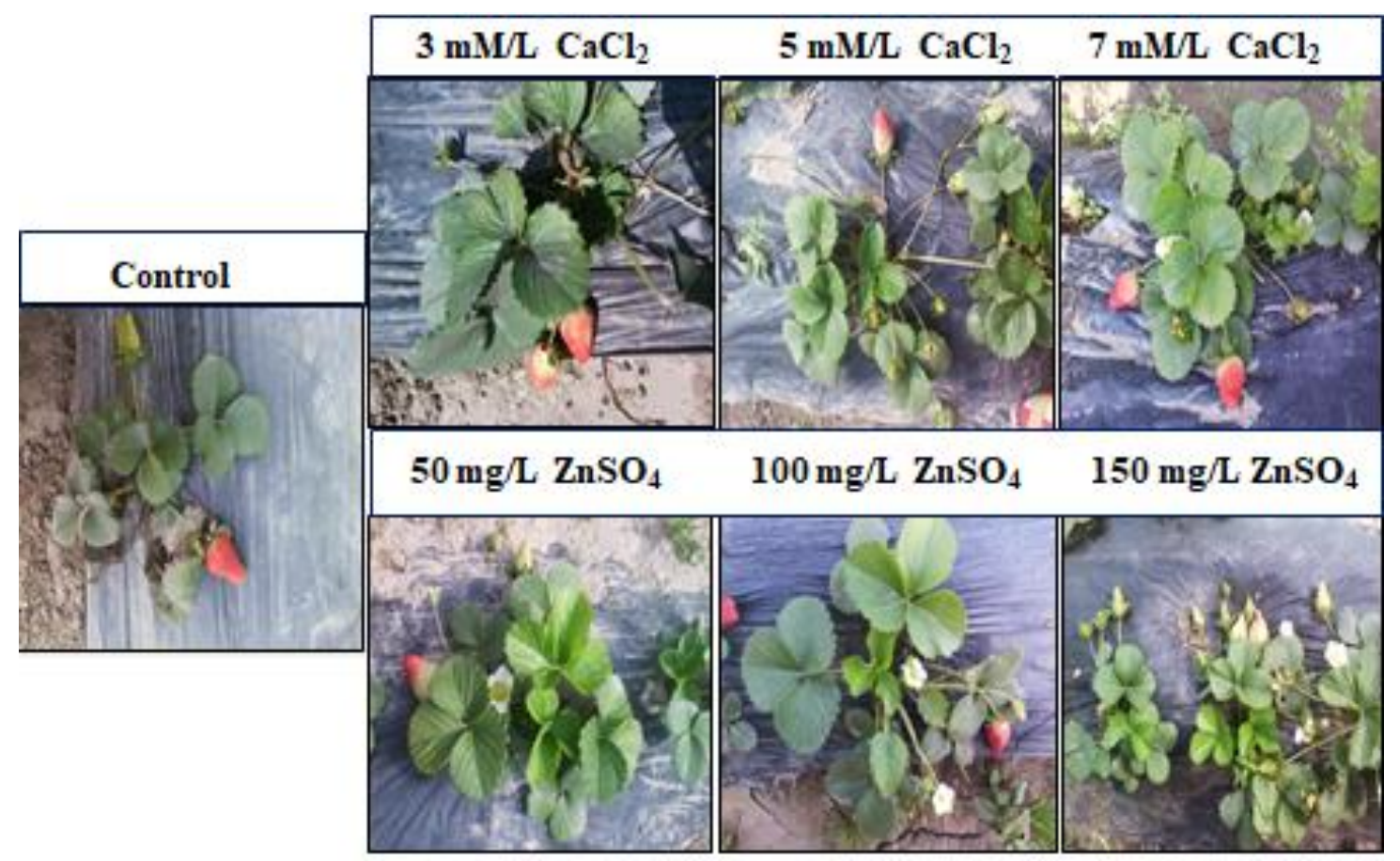

Figure 1. Canopy growth of 'Chandler' strawberry affected by exogenous application of inorganic salts. Treated strawberry plants were healthier as compared to control plants

\section{Yield parameters}

Statistical analysis regarding strawberry marketable yield demonstrated significant increased results (Table 2). Marketable yield was increased with exogenous application 
of salts during growing season. Maximum marketable yield (348.5 $\mathrm{g}$ plant $^{-1}$ ) was observed from strawberry plants those were sprayed with $7 \mathrm{mM} / \mathrm{L} \mathrm{CaCl}_{2}$ followed by other treatments including $100 \mathrm{mg} / \mathrm{L} \mathrm{ZnSO}_{4}\left(319.0 \mathrm{~g} \mathrm{plant}^{-1}\right), 150 \mathrm{mg} / \mathrm{L} \mathrm{ZnSO} 4$ $\left(285.0 \mathrm{~g} \mathrm{plant}^{-1}\right), 5 \mathrm{mM} / \mathrm{L} \mathrm{CaCl}_{2}\left(218.2 \mathrm{~g} \mathrm{plant}^{-1}\right), 3 \mathrm{mM} / \mathrm{L} \mathrm{CaCl}_{2}\left(177.2 \mathrm{~g} \mathrm{plant}^{-1}\right)$ and $50 \mathrm{mg} / \mathrm{L} \mathrm{ZnSO}_{4}$ (159.7 $\mathrm{g} \mathrm{plant}^{-1}$ ), respectively while minimum marketable yield $\left(156.7 \mathrm{~g} \mathrm{plant}^{-1}\right)$ was found with control treatment. It was noted that higher concentration of $\mathrm{CaCl}_{2}(7 \mathrm{mM} / \mathrm{L})$ and medium concentration of $\mathrm{ZnSO}_{4}(100 \mathrm{mg} / \mathrm{L})$ found more responsive for increasing marketable yield however when $\mathrm{ZnSO}_{4}$ concentration was increased from $100 \mathrm{mg} / \mathrm{L}$ decreasing trend was observed. Unmarketable yield (40.5 $\left.\mathrm{g} \mathrm{plant}^{-1}\right)$ was also reduced with $7 \mathrm{mM} / \mathrm{L} \mathrm{CaCl} \mathrm{Ca}_{2}$ while maximum unmarketable yield (107.0 $\left.\mathrm{g} \mathrm{plant}^{-1}\right)$ was observed with control treatment. It was suggested that foliar application of $\mathrm{CaCl}_{2}(7 \mathrm{mM} / \mathrm{L})$ was more effective for increasing the marketable yield and to reduced unmarketable yield. Small size yield was also influenced with $7 \mathrm{mM} / \mathrm{L} \mathrm{CaCl}_{2}$ application and decreasing trend was observed in all treated strawberry plants while maximum yield of small sized strawberries (84.0 g plant $\left.^{-1}\right)$ were observed in control treatment (Table 2).

Table 1. Effect of exogenous application of inorganic salts on vegetative growth of strawberry plants $c v$. 'Chandler' Mean $\pm S E$

\begin{tabular}{|c|c|c|c|c|c|}
\hline Treatments & $\begin{array}{l}\text { No. of leaves } \\
\left.\text { (plant }^{-1}\right)\end{array}$ & $\begin{array}{l}\text { Leaf area } \\
\left(\mathrm{cm}^{2}\right)\end{array}$ & $\begin{array}{c}\text { Flower anthesis } \\
\text { (days) }\end{array}$ & $\begin{array}{l}\text { No. of crowns } \\
\left(\text { plant }^{-1}\right)\end{array}$ & $\begin{array}{l}\text { No. of runners } \\
\quad\left(\text { plant }^{-1}\right)\end{array}$ \\
\hline Control & $8.00 \pm 1.08 \mathrm{~g}$ & $27.50 \pm 1.04 \mathrm{~g}$ & $42.25 \pm 0.48 \mathrm{a}$ & $2.50 \pm 0.29 \mathrm{~g}$ & $3.50 \pm 0.29 \mathrm{a}$ \\
\hline $3 \mathrm{mM} / \mathrm{L} \mathrm{CaCl} 2$ & $12.00 \pm 0.41 \mathrm{e}$ & $35.00 \pm 0.41 \mathrm{e}$ & $41.50 \pm 0.65 \mathrm{a}$ & $3.25 \pm 0.25 \mathrm{f}$ & $3.75 \pm 0.25 \mathrm{a}$ \\
\hline $5 \mathrm{mM} / \mathrm{L} \mathrm{CaCl} 2$ & $13.50 \pm 0.29 \mathrm{~d}$ & $39.50 \pm 1.71 \mathrm{~d}$ & $41.25 \pm 0.63 \mathrm{a}$ & $4.50 \pm 0.29 \mathrm{~d}$ & $3.75 \pm 0.25 \mathrm{a}$ \\
\hline $7 \mathrm{mM} / \mathrm{L} \mathrm{CaCl}_{2}$ & $18.25 \pm 0.25 \mathrm{a}$ & $47.00 \pm 1.08 \mathrm{a}$ & $40.00 \pm 0.71 \mathrm{a}$ & $7.50 \pm 0.29 \mathrm{a}$ & $3.80 \pm 0.41 \mathrm{a}$ \\
\hline $50 \mathrm{mg} / \mathrm{L} \mathrm{ZnSO}_{4}$ & $10.75 \pm 0.48 \mathrm{f}$ & $34.50 \pm 0.65 \mathrm{f}$ & $39.75 \pm 0.25 b$ & $3.50 \pm 0.29 \mathrm{e}$ & $3.71 \pm 0.25 \mathrm{a}$ \\
\hline $100 \mathrm{mg} / \mathrm{L} \mathrm{ZnSO}_{4}$ & $15.25 \pm 0.48 b$ & $44.00 \pm 0.91 \mathrm{~b}$ & $27.75 \pm 0.75 \mathrm{~d}$ & $6.50 \pm 0.00 \mathrm{~b}$ & $3.78 \pm 0.25 \mathrm{a}$ \\
\hline $150 \mathrm{mg} / \mathrm{L} \mathrm{ZnSO}{ }_{4}$ & $14.50 \pm 0.29 \mathrm{c}$ & $42.75 \pm 1.38 \mathrm{c}$ & $33.50 \pm 0.29 \mathrm{c}$ & $5.50 \pm 0.29 \mathrm{c}$ & $3.75 \pm 0.29 \mathrm{a}$ \\
\hline $\operatorname{LSD}(P \leq 0.05)$ & 1.25 & 2.32 & 1.69 & 0.79 & 0.79 \\
\hline C.V. \% & 6.2 & 4.4 & 2.92 & 11.43 & 19.05 \\
\hline
\end{tabular}

Treatments means which represent same letter are statistically non-significant $(p>0.05)$

Table 2. Effect of exogenous application of inorganic salts on yield of strawberry cv. 'Chandler' Mean \pm SE

\begin{tabular}{c|c|c|c}
\hline Treatments & $\begin{array}{c}\text { Marketable } \\
\left(\text { g plant }^{-1}\right)\end{array}$ & $\begin{array}{c}\text { Unmarketable } \\
\left(\text { g plant }^{-1}\right)\end{array}$ & $\begin{array}{c}\text { Small size } \\
\left(\text { g plant }^{-1}\right)\end{array}$ \\
\hline Control & $156.7 \pm 1.31 \mathrm{~g}$ & $107.0 \pm 3.49 \mathrm{a}$ & $84.0 \pm 2.27 \mathrm{a}$ \\
$3 \mathrm{mM} / \mathrm{L} \mathrm{CaCl}_{2}$ & $177.2 \pm 8.33 \mathrm{e}$ & $92.2 \pm 1.11 \mathrm{~b}$ & $65.0 \pm 1.47 \mathrm{~b}$ \\
$5 \mathrm{mM} / \mathrm{L} \mathrm{CaCl}_{2}$ & $218.2 \pm 4.23 \mathrm{~d}$ & $72.2 \pm 1.11 \mathrm{~d}$ & $54.2 \pm 1.11 \mathrm{~d}$ \\
$7 \mathrm{mM} / \mathrm{L} \mathrm{CaCl}_{2}$ & $348.5 \pm 7.08 \mathrm{a}$ & $40.5 \pm 1.55 \mathrm{~g}$ & $38.0 \pm 1.29 \mathrm{~g}$ \\
$50 \mathrm{mg} / \mathrm{L} \mathrm{ZnSO}_{4}$ & $159.7 \pm 12.97 \mathrm{f}$ & $90.0 \pm 0.41 \mathrm{c}$ & $63.7 \pm 0.85 \mathrm{c}$ \\
$100 \mathrm{mg} / \mathrm{L} \mathrm{ZnSO}_{4}$ & $319.0 \pm 15.03 \mathrm{~b}$ & $54.7 \pm 1.60 \mathrm{f}$ & $41.7 \pm 0.48 \mathrm{f}$ \\
$150 \mathrm{mg} / \mathrm{L} \mathrm{ZnSO} 4$ & $285.0 \pm 14.30 \mathrm{c}$ & $65.5 \pm 1.94 \mathrm{e}$ & $53.0 \pm 0.91 \mathrm{e}$ \\
\hline $\mathrm{LSD}(P \leq 0.05)$ & 36.28 & 6.59 & 4.42 \\
C.V. \% & 9.65 & 5.2 & 4.56 \\
\hline
\end{tabular}

Treatments means which represent same letter are statistically non-significant $(p>0.05)$ 


\section{Fruit quality parameters}

\section{Firmness}

Statistical analysis of strawberry fruit firmness affected by foliar application of salts demonstrated significant differences among different treatment means. Maximum firmness $\left(0.96 \mathrm{~kg} . \mathrm{cm}^{-2}\right)$ was achieved in fruits where plants were sprayed with $7 \mathrm{mM} / \mathrm{L}$ $\mathrm{CaCl}_{2}$ followed by plants sprayed with different concentrations of $\mathrm{CaCl}_{2}$ and $\mathrm{ZnSO}_{4}$ while, less firmness $\left(0.42 \mathrm{~kg}\right.$. $\left.\mathrm{cm}^{-2}\right)$ was observed in control fruits (Fig. 2). Calcium chloride as firming agent was proved better for enhancing strawberry firmness when sprayed on plants during growth stages.

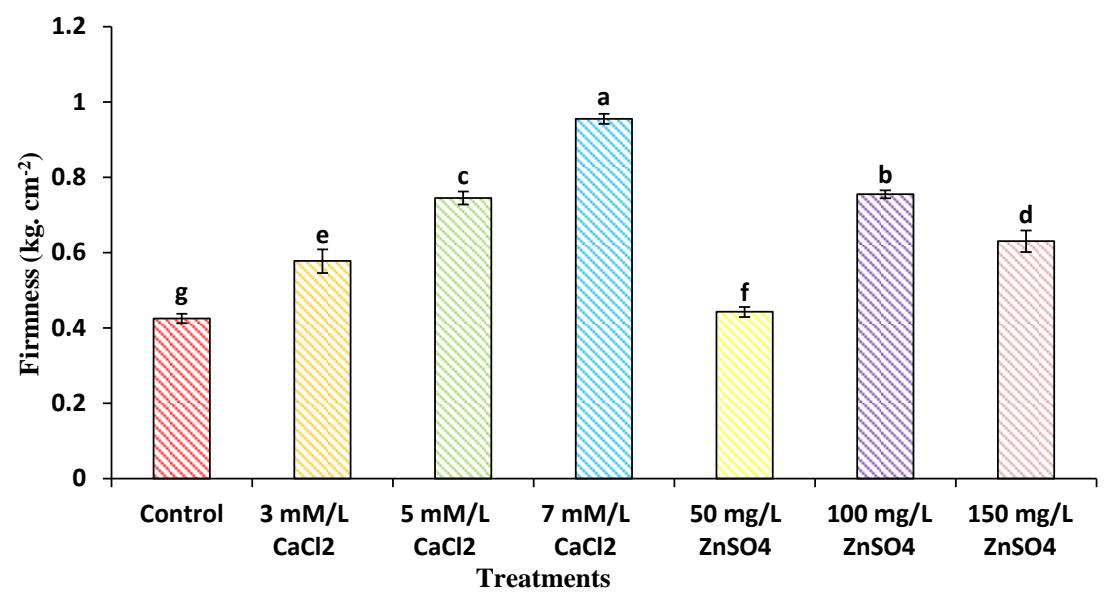

Figure 2. Effect of exogenous application of inorganic salts on firmness $\left(\mathrm{kg}^{\left.\mathrm{c} \mathrm{cm}^{-2}\right)}\right.$ of strawberry fruit cv. 'Chandler'. Vertical bars represent $\pm S E$ of means

\section{Total soluble solids}

Total soluble solids increased in those strawberry fruits where plants were treated with different concentrations of $\mathrm{CaCl}_{2}$ and $\mathrm{ZnSO}_{4}$. Maximum amount of total soluble solids $\left(8.2^{\circ}\right.$ Brix) were observed with $7 \mathrm{mM} / \mathrm{L} \mathrm{CaCl}_{2}$ application followed by other treatments including $100 \mathrm{mg} / \mathrm{L} \mathrm{ZnSO}_{4}\left(7.5^{\circ} \mathrm{Brix}\right), 5 \mathrm{mM} / \mathrm{L} \mathrm{CaCl}_{2}\left(7.1^{\circ} \mathrm{Brix}\right), 150 \mathrm{mg} / \mathrm{L}$ $\mathrm{ZnSO}_{4}\left(6.8{ }^{\circ} \mathrm{Brix}\right), 3 \mathrm{mM} / \mathrm{L} \mathrm{CaCl}_{2}\left(6 .{ }^{\circ}{ }^{\circ}\right.$ Brix $)$ and $50 \mathrm{mg} / \mathrm{L} \mathrm{ZnSO}_{4}$ (5.9 $\left.{ }^{\circ} \mathrm{Brix}\right)$, respectively while minimum TSS contents $\left(5.7^{\circ} \mathrm{Brix}\right)$ were observed in control treatment (Fig. 3a).

\section{Titratable acidity}

Non-significant trend was recorded regarding effect of foliar spray of $\mathrm{CaCl}_{2}$ and $\mathrm{ZnSO}_{4}$ on acid contents of strawberry fruit. Quantitatively acid contents were found maximum in control treatment as compared to fruits of treated plants but statistically there was no difference between different treatment means (Fig. 3b).

\section{TSS:TA ratio}

TSS:TA ratio is major indicator of quality which determines the shelf life of fruit. Statistical analysis regarding strawberry fruit TSS:TA ratio showed significant increased trend. Maximum TSS:TA ratio (7.62) was observed in strawberry fruits where plants were treated with $7 \mathrm{mM} / \mathrm{L} \mathrm{CaCl}_{2}$ while minimum TSS:TA ratio (4.48) was 
observed in control treatment (Fig. 3c). Increasing trend in TSS:TA ratio was found in treated fruits due maximum TSS contents and minimum acid contents however, in control fruits decreasing trend was found due to maximum acid contents and minimum TSS contents.

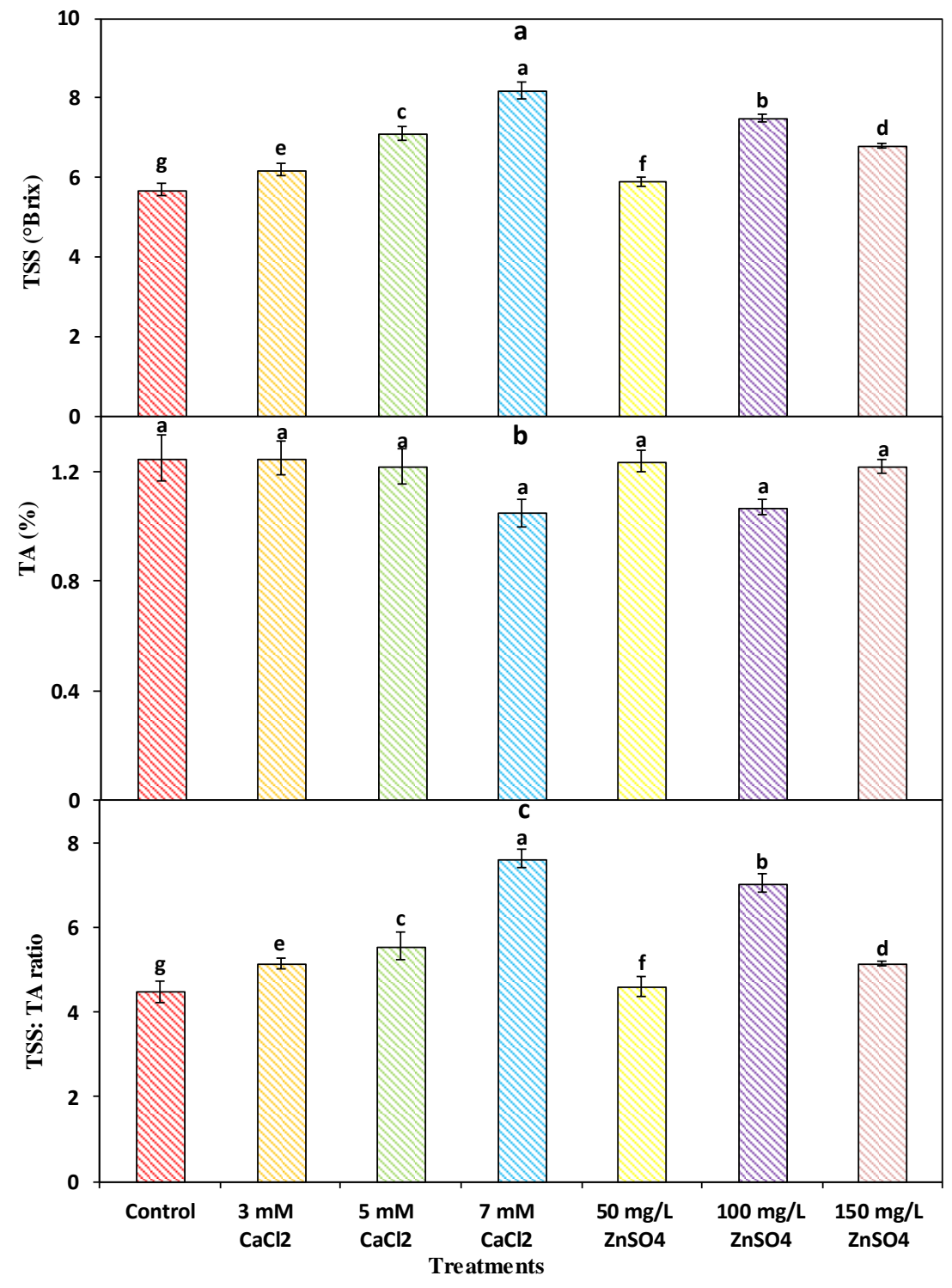

Figure 3. Effect of exogenous application of inorganic salts on (a) total soluble solids, (b) titratable acidity and (c) TSS:TA ratio of strawberry fruit $c v$. 'Chandler'. Vertical bars represent $\pm S E$ of means

\section{Vitamin $C$}

Statistically significant increased trend was noted regarding vitamin $\mathrm{C}$ contents of strawberry fruit. Maximum (55.69 mg $100 \mathrm{~g} \mathrm{~g}^{-1}$ ) values were observed in strawberry fruits where plants were treated with $7 \mathrm{mM} / \mathrm{L} \mathrm{CaCl}_{2}$ followed by other treatments including 100 $\mathrm{mg} / \mathrm{L} \mathrm{ZnSO} 4\left(53.30 \mathrm{mg} 100 \mathrm{~g}^{-1}\right), 5 \mathrm{mM} / \mathrm{L} \mathrm{CaCl}_{2}$ (46.86 mg $\left.100 \mathrm{~g}^{-1}\right), 150 \mathrm{mg} / \mathrm{L} \mathrm{ZnSO}_{4}$ (43.29 mg $\left.100 \mathrm{~g}^{-1}\right), 3 \mathrm{mM} / \mathrm{L} \mathrm{CaCl}_{2}\left(41.16 \mathrm{mg} 100 \mathrm{~g}^{-1}\right)$ and $50 \mathrm{mg} / \mathrm{L} \mathrm{ZnSO}_{4}(40.77 \mathrm{mg}$ $\left.100 \mathrm{~g}^{-1}\right)$, respectively while minimum value $\left(35.55 \mathrm{mg} 100 \mathrm{~g}^{-1}\right)$ was observed in control 
treatment. Similar to other parameters the effect of exogenous application of salts was more effective for increasing vitamin C contents of strawberry fruit (Fig. 4a).

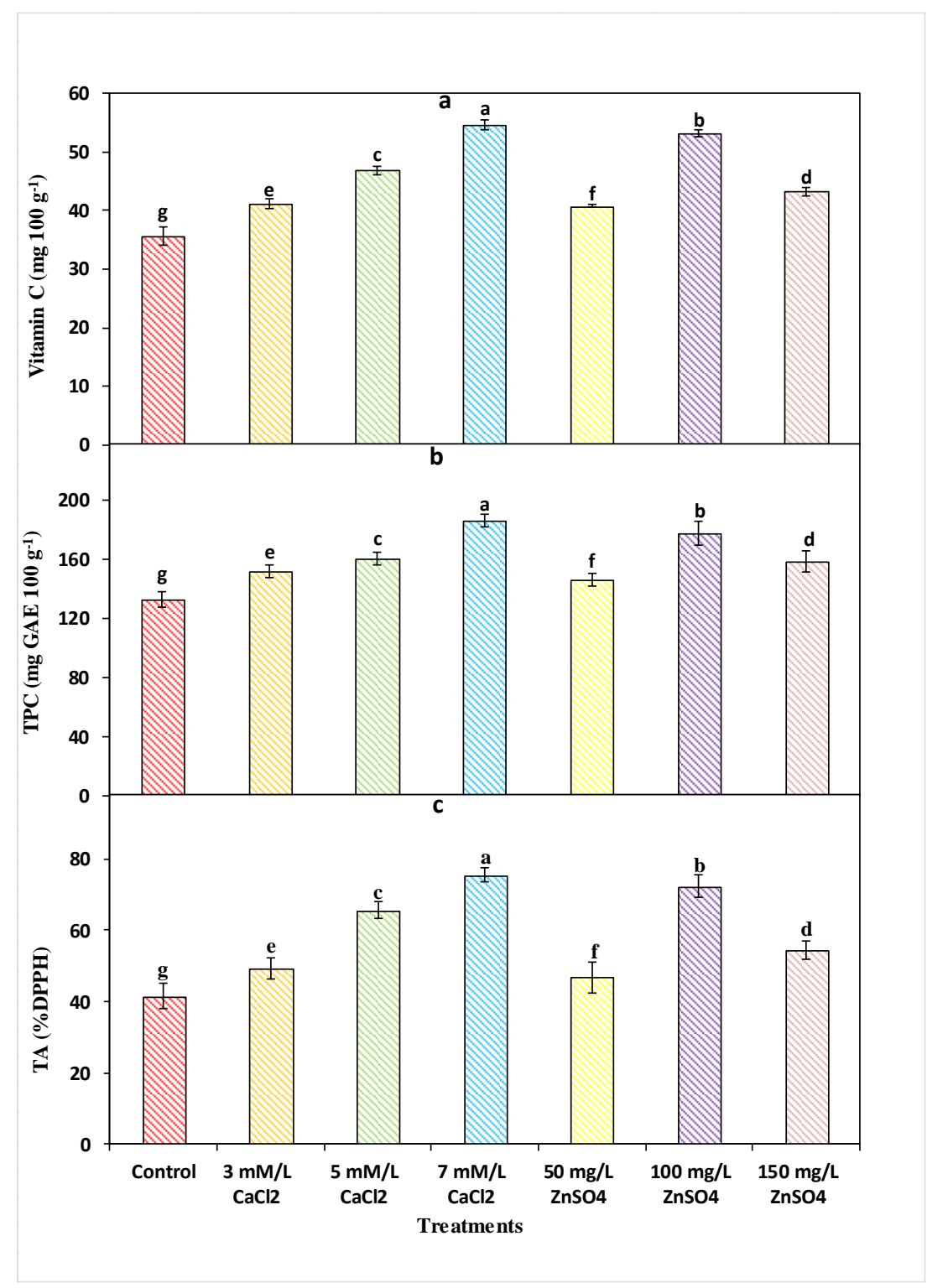

Figure 4. Effect of exogenous application of inorganic salts on (a) vitamin $C$ contents (b) total phenolic contents and (c) total antioxidants of strawberry fruit cv. 'Chandler'. Vertical bars represent $\pm S E$ of means

\section{Total phenolic contents}

Total phenolic contents of strawberry fruit highly influenced with application of salts during growth stages. Increasing trend was noticed with $7 \mathrm{mM} / \mathrm{L} \mathrm{CaCl}_{2}$ and showed higher TPC (186.5 mg GAE $100 \mathrm{~g}^{-1}$ ) value followed by other treatments including $100 \mathrm{mg} / \mathrm{L} \mathrm{ZnSO}_{4}\left(177.5 \mathrm{mg}\right.$ GAE $\left.100 \mathrm{~g}^{-1}\right), 5 \mathrm{mM} / \mathrm{L} \mathrm{CaCl} 2\left(160.5 \mathrm{mg}\right.$ GAE $\left.100 \mathrm{~g}^{-1}\right)$, $150 \mathrm{mg} / \mathrm{L} \mathrm{ZnSO}_{4}\left(158.5 \mathrm{mg} \mathrm{GAE} 100 \mathrm{~g}^{-1}\right), 3 \mathrm{mM} / \mathrm{L} \mathrm{CaCl}_{2}\left(151.5 \mathrm{mg} \mathrm{GAE} 100 \mathrm{~g}^{-1}\right)$ and $50 \mathrm{mg} / \mathrm{L} \mathrm{ZnSO}_{4}\left(146.5 \mathrm{mg} \mathrm{GAE} 100 \mathrm{~g}^{-1}\right.$ ), respectively (Fig. 4b). Decreasing trend was noticed with control treatment and observed lower TPC (132.75 mg GAE $\left.100 \mathrm{~g}^{-1}\right)$ value. 


\section{Total antioxidants}

Statistical results regarding total antioxidants in strawberry fruit affected by exogenous application of salts showed significant increased trend. Higher antioxidants $(75.5 \% \mathrm{DPPH})$ were noted in strawberry fruits where plants were treated with $7 \mathrm{mM} / \mathrm{L}$ $\mathrm{CaCl}_{2}$ application. The response of exogenous application of $\mathrm{CaCl}_{2}$ and $\mathrm{ZnSO}_{4}$ was highly effective for increasing the antioxidants in strawberry fruit while minimum (41.5\% DPPH) values were observed in control treatment (Fig. 4c).

\section{Activities of anti-oxidative enzymes}

Enzymatic activities were also influenced with exogenous application of salts (Table 3). While non-significant trend was found regarding catalase activity of strawberry fruit. Quantitatively higher CAT activity was observed in fruits of control treatment as compared to other treatments. Maximum SOD activity $\left(30.2 \mathrm{U} \mathrm{mg}^{-1}\right.$ protein) was observed in strawberry fruits where plants were sprayed with $100 \mathrm{mg} / \mathrm{L}$ $\mathrm{ZnSO}_{4}$ followed by other treatments including $7 \mathrm{mM} / \mathrm{L} \mathrm{CaCl}_{2}\left(26.4 \mathrm{U} \mathrm{mg}^{-1}\right.$ protein), $150 \mathrm{mg} / \mathrm{L} \mathrm{ZnSO}_{4}\left(24.4 \mathrm{U} \mathrm{mg}^{-1}\right.$ protein), $5 \mathrm{mM} / \mathrm{L} \mathrm{CaCl}_{2}$ (22.3 $\mathrm{U} \mathrm{mg}^{-1}$ protein), $50 \mathrm{mg} / \mathrm{L}$ $\mathrm{ZnSO}_{4}\left(20.1 \mathrm{U} \mathrm{mg}^{-1}\right.$ protein) and $3 \mathrm{mM} / \mathrm{L} \mathrm{CaCl}_{2}\left(19.2 \mathrm{U} \mathrm{mg}^{-1}\right.$ protein), respectively while lower SOD activity (18.0 $\mathrm{U} \mathrm{mg}^{-1}$ protein) was noted in control treatment. It was exhibited that POD activity (1.05 $\mathrm{U} \mathrm{mg}^{-1}$ protein) was decreased with foliar application of $100 \mathrm{mg} / \mathrm{L} \mathrm{ZnSO}_{4}$ while maximum POD activity $\left(3.89 \mathrm{U} \mathrm{mg}^{-1}\right.$ protein) was observed in fruits of control treatment. From results it was found that exogenous application of $\mathrm{ZnSO}_{4}(100 \mathrm{mg} / \mathrm{L})$ increased the SOD activity in strawberry fruits while POD activity was decreased with this concentration (Table 3).

Table 3. Effect of exogenous application of inorganic salts on enzymatic activities of strawberry fruit $\mathrm{cv}$. 'Chandler' Mean $\pm S E$

\begin{tabular}{|c|c|c|c|}
\hline Treatments & CAT (U mg ${ }^{-1}$ protein) & SOD $\left(\mathrm{U} \mathrm{mg}^{-1}\right.$ protein $)$ & POD $\left(\mathrm{U} \mathrm{mg}^{-1}\right.$ protein) \\
\hline Control & $12.8 \pm 0.01 \mathrm{a}$ & $18.0 \pm 0.11 \mathrm{~g}$ & $3.89 \pm 0.26 \mathrm{a}$ \\
\hline $3 \mathrm{mM} / \mathrm{L} \mathrm{CaCl}_{2}$ & $10.7 \pm 0.01 \mathrm{a}$ & $19.2 \pm 0.12 \mathrm{f}$ & $2.34 \pm 0.03 b$ \\
\hline $5 \mathrm{mM} / \mathrm{L} \mathrm{CaCl}_{2}$ & $10.8 \pm 0.01 \mathrm{a}$ & $22.3 \pm 0.22 \mathrm{~d}$ & $2.16 \pm 0.01 \mathrm{~d}$ \\
\hline $7 \mathrm{mM} / \mathrm{L} \mathrm{CaCl}_{2}$ & $10.6 \pm 0.01 \mathrm{a}$ & $26.4 \pm 0.23 b$ & $1.35 \pm 0.02 \mathrm{f}$ \\
\hline $50 \mathrm{mg} / \mathrm{L} \mathrm{ZnSO}_{4}$ & $10.2 \pm 0.25 \mathrm{a}$ & $20.1 \pm 0.36 \mathrm{e}$ & $2.25 \pm 0.02 \mathrm{c}$ \\
\hline $100 \mathrm{mg} / \mathrm{L} \mathrm{ZnSO} 4$ & $10.0 \pm 0.24 \mathrm{a}$ & $30.2 \pm 0.37 \mathrm{a}$ & $1.05 \pm 0.02 \mathrm{~g}$ \\
\hline $150 \mathrm{mg} / \mathrm{L} \mathrm{ZnSO} 4$ & $10.6 \pm 0.21 \mathrm{a}$ & $24.4 \pm 0.38 \mathrm{c}$ & $1.57 \pm 0.02 \mathrm{e}$ \\
\hline $\operatorname{LSD}(P \leq 0.05)$ & 0.4 & 1.61 & 1.19 \\
\hline C.V. $\%$ & 21.72 & 3.57 & 11 \\
\hline
\end{tabular}

Treatments means which represent same letter are statistically non-significant $(p>0.05)$

\section{Survival}

During whole growing season maximum survival (94.7\%) was observed in those strawberry plants treated with $7 \mathrm{mM} / \mathrm{L} \mathrm{CaCl}_{2}$ followed by other treatments $100 \mathrm{mg} / \mathrm{L}$ $\mathrm{ZnSO}_{4}$ (90.7\%), $150 \mathrm{mg} / \mathrm{L} \mathrm{ZnSO}_{4}(83.5 \%), 5 \mathrm{mM} / \mathrm{L} \mathrm{CaCl}_{2}$ (80.5), $3 \mathrm{mM} / \mathrm{L} \mathrm{CaCl}_{2}$ $(72.2 \%)$ and $50 \mathrm{mg} / \mathrm{L} \mathrm{ZnSO}_{4}(71.5 \%)$, respectively. Minimum plants survival (64.2\%) was observed in control plants. It was suggested that exogenous application of salts is highly effective strategy to enhance survival (\%) of strawberry plants (Fig. 5). 


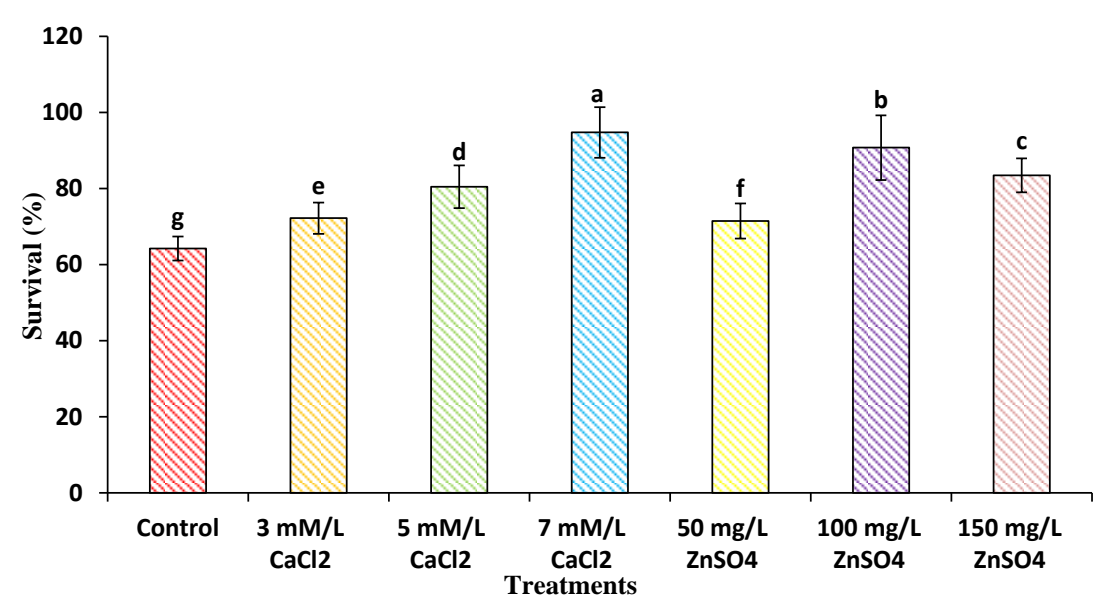

Figure 5. Effect of exogenous application of inorganic salts on survival (\%) of strawberry plants $c v$. 'Chandler'. Vertical bars represent $\pm S E$ of means

\section{Discussion}

Strawberry has fast growing habit which needs sufficient amount of macro and micro nutrients throughout growing season (Medeiros et al., 2015). Foliar application of salts at proper and regular intervals play major role in increasing fruit set and productivity of strawberry plants (Phillips, 2004; Abdollahi et al., 2010). For successful strawberry production calcium is classified as secondary important nutrient for strawberry plant requirement and structural part of cell walls and promotes rapid plant growth (Anonymous, 2014). Zinc is metal compound associated with many enzymes and proteins which played functional role for improving normal plant growth and developmental processes (Nasiri et al., 2010).

In current study, different concentrations of $\mathrm{CaCl}_{2}$ and $\mathrm{ZnSO}_{4}$ were applied through foliar application at different growth stages. Maximum number of leaves, leaf area and number of crowns were found with highest concentration of $\mathrm{CaCl}_{2}(7 \mathrm{mM} / \mathrm{L})$ as compared to other treatments (Table 1). Calcium as important secondary nutrient for strawberry plant growth enhanced the vegetative growth because it improve the inflexibility of plasma membrane of plant cells, most prominent in the apoplast, the cell wall space where it plays major role for cross-link within pectin polysaccharide matrix and contribute their stability (Lester and Grusak, 1999; Anonymous, 2014). The quantity of calcium taken up by the plants from the soil solution, most of it transferred to the leaves but very little amount goes to the fruit (Kadir, 2004). Therefore, for better growth plants need regular supply of calcium for vigorous canopy development (DelAmor and Marcelis, 2003). Our findings regarding foliar application of $\mathrm{CaCl}_{2}$ enhanced the vegetative growth of strawberry plants similar with previous findings where they observed that foliar spray of $\mathrm{CaCl}_{2}(0.6 \%)$ increased the number of leaves, leaf area and improved plant canopy of strawberry (Bakshi et al., 2013). Our results exhibited that strawberry flowering was not affected by $\mathrm{CaCl}_{2}$ application and these results are in line with previous findings where foliar applied $0.3 \% \mathrm{CaCl}_{2}$ not increased the cluster of flowers in tomato crop ( $\mathrm{Rab}$ and Haq, 2012). In this study flower initiation was found earlier with $100 \mathrm{mg} / \mathrm{L} \mathrm{ZnSO}_{4}$ application as compared to other treatments and these results are in accordance with Lolaei et al. (2012) who reported that foliar application of $\mathrm{ZnSO}_{4}$ improved the flowering in strawberry cultivar 'Camarosa'. 
During whole growing season increasing trend was found regarding marketable yield and fruit setting was increased with $7 \mathrm{mM} / \mathrm{L} \mathrm{CaCl} 2$ as compared to other treatments (Table 2). Our results proved that $\mathrm{CaCl}_{2}$ treatments reduced the unmarketable and small size strawberries and increased the marketable yield. The mechanism through which external application of $\mathrm{CaCl}_{2}$ improved plant growth and marketable yield of strawberries, it is because of calcium on plant surface create electrochemical potential gradient which favors inward movement of calcium (Anonymous, 2014). Calcium sequestered into vacuole through transport across the tonoplast, binds to calcium binding proteins (calmodulin) and also with intracellular organelles such as mitochondria, nucleus, endoplasmic reticulum and chloroplast. After binding, it is transmitted to receptor proteins that elicit proper responses to the stimulus (Taiz and Zeiger, 2006). Our results exhibited that marketable yield increased with foliar spray of $\mathrm{CaCl}_{2}$ similar with some previous findings where foliar application of $10 \mathrm{mM} / \mathrm{L} \mathrm{CaCl}_{2}$ improved the yield and qualitative characteristics of strawberry cultivar 'Pajaro' (Kazemi, 2015). The beneficial aspect of $\mathrm{CaCl}_{2}$ for increasing fruit setting and yield was due to maximum capability of photosynthesis and $\mathrm{CaCl}_{2}$ increased the process of hormone metabolism due to that process auxin synthesis increase in strawberry plants which is essential for yield and growth (Bakshi et al., 2013; Kazemi, 2015).

Higher strawberry firmness was achieved with $7 \mathrm{mM} / \mathrm{L} \mathrm{CaCl}_{2}$ application as compared to other treatments (Fig. 2). Firmness quality increased with foliar spray of $\mathrm{CaCl}_{2}$ because calcium spray increased the level of pectin in fruit cells due to that cell wall rigidity increased and fruits nature was more firm (Sams, 1999; Maas, 1998). In previous findings it was confirmed that foliar application of calcium containing products improved firmness of kiwifruit cultivar 'Tsechelidis' as compared with control treatment (Koutinas et al., 2010). Maximum total soluble solids were found in those strawberries which were treated with $\mathrm{CaCl}_{2}(7 \mathrm{mM} / \mathrm{L})$ as compared to other treatments (Fig. 3a). Statistically acid contents were found non-significant but maximum were noted with control treatment (Fig. 3b). In this experiment it was noted that calcium and zinc foliar sprays decrease the acid contents which resulted in increased total soluble solids contents of fruit. Similar findings were reported by Ahlawat et al. (1985) in grapes. Maximum TSS:Acid ratio was also observed in strawberries where plants treated with $7 \mathrm{mM} / \mathrm{L} \mathrm{CaCl}_{2}$ as compared to others (Fig. 3c). The balance between TSS:Acid ratio was major indication of strawberry ripening and maturity.

Vitamin $\mathrm{C}$ known as ascorbic acid acted as an antioxidant which increased the nutritional value of fruit (Rapisarda et al., 2008). Higher contents of vitamin $\mathrm{C}$ were observed with $7 \mathrm{mM} / \mathrm{L} \mathrm{CaCl} 2$ application (Fig. $4 a$ ). Vitamin $\mathrm{C}$ increased with $\mathrm{CaCl}_{2}$ application because calcium spray enhanced the activity of several catalytic enzymes which played major role in biosynthesis of vitamin C contents (Kadir, 2004). Over all, fruit quality was increased because calcium spray played major role in plant cell wall integrity and improved the nutritional status of fruits. Calcium deficiency in fruits showed less resistance against pathogens and increased the chances of disease occurrence in plants, so calcium spray suggested as for disease management (Ghani et al., 2011; Naradisorn, 2013). Kazemi (2014) also observed similar findings regarding foliar application of $\mathrm{CaCl}_{2}(10 \mathrm{mM} / \mathrm{L})$ enhanced the quality of strawberry. In literature it was proved that foliar spray of $\mathrm{CaCl}_{2}(0.6 \%)$ increased the TSS contents, ascorbic acid contents of strawberry and lower acidity than control (Bakshi et al., 2013).

In current study, it was observed that application of $\mathrm{CaCl}_{2}$ significantly increased the phenolic compounds and antioxidants in strawberry. Maximum phenolic contents and 
antioxidants were found with $7 \mathrm{mM} / \mathrm{L} \mathrm{CaCl}_{2}$ application as compared to others (Fig. $4 b$, $4 c$ ). External application of calcium activated environmental signals against biotic and abiotic stresses (high light intensity, day length, extreme temperatures, drought, osmotic stress and attack of pathogens) in cell membranes that catalase rapid calcium influx to cytosol and enhanced cytosolic free calcium concentration which enhanced the phenolic compounds and antioxidants in plants (Taiz and Zeiger, 2006; Ali et al., 2013).

Enzymatic activities were influenced with $\mathrm{ZnSO}_{4}$ treatments. Our results suggested that higher catalase and peroxidase activities were observed in control treatment as compared to other treatments (Table 3). Higher catalase activity leading towards senescence and higher peroxidase activity caused off flavor in strawberry fruits. Maximum SOD activity was noted in strawberry fruits where plants were treated with $100 \mathrm{mg} / \mathrm{L} \mathrm{ZnSO}_{4}$ while lower activity was observed in control treatment. In some previous studies it was exhibited that reactive oxygen species (ROS) produced during biochemical changes in strawberry such as hydrogen peroxide and hydroxyl radical which cause oxidative damage (Jimenez et al., 2003). These (ROS) species caused early fruit ripening and led to senescence. Activities of various antioxidant enzymes (CAT, SOD and POD) produce during different growth stages of strawberry fruit and increase the defense response against these ROS species (Anand et al., 2009). Zinc played major role in defense mechanism of plant by increasing activities of antioxidant enzymes and also the regulation process of those genes (chitinase) which required for creating resistance against environmental stresses in plants (Marschner, 1995; Cakmak, 2000).

According to our results it is clear that maximum survival was achieved with $7 \mathrm{mM} / \mathrm{L} \mathrm{CaCl}_{2}$ application while, the other treated plants also respond better for achieving maximum survival as compared to control plants (Fig. 5). Previous studies showed that calcium signaling played critical role in plant resistance to diseases; create defense mechanism against insects and acclimatization against non-biological stresses (Sun et al., 2009). Due to that enzymatic activities increased which enhanced the survival mechanism of strawberry plants. During whole growing season treated strawberry plants showed better response as compared with untreated plants.

\section{Conclusion and future recommendations}

Calcium as secondary important nutrient for strawberry plant plays major role in growth and development. In current study $7 \mathrm{mM} / \mathrm{L} \mathrm{CaCl}_{2}$ treatment was proved best for improving the number of leaves, leaf area and number of crowns while early flower initiation was observed with $100 \mathrm{mg} / \mathrm{L} \mathrm{ZnSO}_{4}$ application. Marketable yield, fruit quality and survival mechanism of strawberry plants maximized with $7 \mathrm{mM} / \mathrm{L} \mathrm{CaCl} 2$ application. However, enzymatic activities were highly influenced with $100 \mathrm{mg} / \mathrm{L}$ $\mathrm{ZnSO}_{4}$ application. By comparison, it is concluded that exogenous application of $7 \mathrm{mM} / \mathrm{L} \mathrm{CaCl} 2$ during growth stages is highly effective strategy for improving marketable yield and quality.

In future combined application of $\mathrm{CaCl}_{2}$ and $\mathrm{ZnSO}_{4}$ should be applied for improving maximum marketable yield and quality. More work is needed on other strawberry cultivars such as Douglas, Toro, Pocahontas, Honeyo and Tufts by using salts to increase the marketable yield and to improve quality attributes.

Acknowledgments. We pay thanks to Ayub Agricultural Research Institute (AARI) for providing the research area for field experiment and special thanks to Institute of Horticultural Sciences and 
Biochemistry Department of University of Agriculture Faisalabad for fruit quality analysis. This paper is extracted from the $\mathrm{PhD}$ dissertation of the first author. The first author thankfully acknowledges the University of Agriculture Faisalabad for providing Ph.D. fellowship scholarship for financial support.

\section{REFERENCES}

[1] Abdollahi, M., Eshghi, S., Tafazzoli, E., Moosavi, N. (2010): Effects of paclobutrazol, boric acid and zinc sulfate on vegetative and reproductive growth of strawberry cv. Selva. - J. Agr. Sci. Tech. 14: 357-363.

[2] Adda-Bjarnadottir, M. (2012): Strawberries. Nutrition facts and health benefits. https://authoritynutrition.com/foods/strawberries/.

[3] Ahlawat, V. P., Sharma, S., Dahia, S. S., Yamdagni, R. (1985): Effect of iron sprays on physico-chemical characteristics of grapes cv. Beauty Seedless. - Progressive Horticulture 17: 100-2.

[4] Ainsworth, E. A., Gillespie, K. M. (2007): Estimation of total phenolic contents and other oxidation substances in plant tissue using Folin-Ciocalteu reagent. - Nature Protocols. 2: 875-877.

[5] Ali, S., Masud, T., Abbasi, K. S., Mahmood, T., Hussain, I. (2013): Influence of $\mathrm{CaCl}_{2}$ on biochemical composition, antioxidant and enzymatic activity of apricot at ambient storage. - Pak. J. Nutr. 12: 476-483.

[6] Alshaal, T., El-Ramady, H. R. (2017): Foliar application: from plant nutrition to Biofortification. - Env. Biodiv. Soil Security 1: 71-83.

[7] Anand, T., Ghaskaran, R., Raguchander, T., Samiyappan, R., Prakasan, V., Gopalakrishnan, C. (2009): Defence responses of chilli fruits to Colletotrichum capsici and Alternaria alternata. - Biol. Plant. 53: 553-559.

[8] Anonymous (2014): Haifa. Strawberry crop guide: mineral nutrition of strawberries. http://www.haifagroup.com/knowledge_center/crop_guides/strawberry/mineral_nutrition _of_strawberries/.

[9] Anonymous (2018): Growing produce. Why foliar feeding? - https://www.Growing produce.com/sponsor/yara/why-foliar-feeding/.

[10] Asad, A. (1997): Strawberry production and marketing potentials. - Advisory Leaflet of MFVDP 30: 1-2.

[11] Bakshi, P., Jasrotia, A., Wali, V. K., Sharma, A., Bakshi, M. (2013): Influence of preharvest application of calcium and micro-nutrients on growth, yield, quality and shelf-life of strawberry cv. Chandler. - Indian. J. Agr. Sci. 83(8): 831-835.

[12] Bramlage, W. G., Drake, M., Weis, S. A. (1985): Comparisons of calcium chloride, calcium phosphate and a calcium chelate as foliar sprays for 'McIntosh' apple trees. - J. Am. Soc. Hortic. Sci. 110: 786-789.

[13] Brand-Williams, W., Cuvelier, M. E., Berset, C. (1995): Use of free radical method to evaluate antioxidant activity. - Lebensm. Wiss. Technol. 28: 25-30.

[14] Cakmak, I. (2000): Possible roles of zinc in protecting plant cells from damage by reactive oxygen species. - The New Phytologist 146(2): 185-205.

[15] Cheour, F., Willemot, C., Arul, J., Desjardin, Y., Makhlouf, J., Charest, P. M., Gosselin, A. (1990): Foliar application of calcium chloride delays postharvest ripening of strawberry. - J. Am. Soc. Hortic. Sci. 115: 789-792.

[16] Del-Amor, F. K., Marcelis, L. F. M. (2003): Regulation of nutrient uptake, water uptake and growth under calcium starvation and recovery. - J. Hort. Sci. Biotechnol. 78: 343349.

[17] Elad, Y., Volpin, H. (1993): Reduced development of grey mould (Botrytis cinerea) in bean and tomato plants by calcium nutrition. - J. Phytopathol. 139: 146-156. 
[18] Ghani, M. A. A., Awang, Y., Sijam, K. (2011): Disease occurrence and fruit quality of pre-harvest calcium treated red flesh dragon fruit (Hylocereusm polyrhizus). - Afr. J. Biotech. 10(9): 1550-1558.

[19] GOP (2015): Fruits, Vegetables and Condiments Statistics of Pakistan. - Government of Pakistan, Ministry of National Food Security and Research, Islamabad.

[20] Jimenez, A., Gomez, F. J. M., Llanos, M. R., Sevilla, F. (2003): Antioxidant systems and their relationship with the response of pepper fruits to storage at $20^{\circ} \mathrm{C}$. $-\mathrm{J}$. Agr. Food Chem. 51: 6293-6299.

[21] Kadir, S. A. (2004): Fruit quality at harvest of 'Jonathan' apple treated with foliar applied calcium chloride. - J. Plant. Nut. 27: 1991-2006.

[22] Kazemi, M. (2014): Influence of foliar application of iron, calcium and zinc sulfate on vegetative growth and reproductive characteristics of strawberry cv.'Pajaro'. - Trakia J. Sci. 1: 21-26.

[23] Kazemi, M. (2015): Effect of iron (Fe-EDDHA), calcium chloride and zinc sulphate on vegetative growth, yield and fruit quality of strawberry (Fragaria $\times$ Ananassa Duch. cv. Pajaro). - Jordan J. Agr. Sci. 173(3643): 1-8.

[24] Koutinas, N., Sotiropoulos, T., Petridis, A., Almaliotis, D., Deligeorgis, E., Therios, I., Voulgarakis, N. (2010): Effects of preharvest calcium foliar sprays on several fruit quality attributes and nutritional status of the kiwifruit cultivar 'Tsechelidis'. HortScience 45(6): 984-987.

[25] Lester, G. E., Grusak, M. A. (1999): Postharvest application of calcium and magnesium to honeydew and netted muskmelons: effects on tissue ion concentrations, quality and senescence. - J. Amer. Soc. Hort. Sci. 124: 545-552.

[26] Li, H., Huang, R., Li, T., Hu, K. (2010): Ability of nitrogen and phosphorus assimilation of seven strawberry cultivars in a northern Atlantic coastal soil. - World Congress of Soil Science, Soil Solutions for a Changing World (Vol. 19). 1 - 6 August 2010, Brisbane, Australia.

[27] Liu, D., Zou, J., Meng, Q., Zou, J., Jiang, W. (2009): Uptake and accumulation and oxidative stress in garlic (Allium sativum L.) under lead phytotoxicity. - Ecotoxicol. 18: 134-143.

[28] Lolaei, A., Rezaei, M. A., Khorrami, M., Kaviani, B. (2012): Effect of paclobutrazol and sulfate zinc on vegetative growth, yield and fruit quality of strawberry (Fragaria $\times$ ananassa Duch. cv. Camarosa). - Ann. Biol. Res. 3(10): 4657-4662.

[29] Maas, J. L. (1998): Compendium of Strawberry Diseases. - APS Press, St. Paul, MN.

[30] Mabood, M. (1994): The future prospects of strawberry production in Pakistan. Advisory Leaflet 17: 51-52.

[31] Marschner, H. (1995): Mineral Nutrition of Higher Plants. $2^{\text {nd }}$ Ed. - Academic Press, London.

[32] Medeiros, R. F., Pereira, W. E., Rodrigues, R. D. M., Nascimento, R. D., Suassuna, J. F., Dantas, T. A. (2015): Growth and yield of strawberry plants fertilized with nitrogen and phosphorus. - Rev. Bras. Eng. Agr. Amb. 19: 865-870.

[33] Meena, D., Tiwari, R., Singh, O. P. (2014): Effect of nutrient spray on growth, fruit yield and quality of aonla. - Ann. Plant Soil Res. 16(3): 242-245.

[34] Meier, U. (2006): A note on the power of Fisher's least significant difference procedure. - Pharmaceutical Statistics: J. App. Stat. 5(4): 253-263.

[35] Naradisorn, M. (2013): Effect of calcium nutrition on fruit quality and post-harvest diseases. - Int. J. Sci. Innovations and Discoveries 3(1): 8-13.

[36] Nasiri, Y., Zehtab-Salmasi, S., Nasrullahzadeh, S., Najafi, N., Ghassemi-Golezani, K. (2010): Effects of foliar application of micronutrients (Fe and $\mathrm{Zn}$ ) on flower yield and essential oil of chamomile (Matricaria chamomilla L.). - J. Med Plant. Res. 4: 17331737. 
[37] Phillips, M. (2004): Economic benefit from using micronutrients for the farmer and the fertilizer producer. - IFA. International Symposium on Micronutrients, Feb 23-24, 2004, New Delhi.

[38] Qureshi, K. M., Chughtai, S., Qureshi, U. S., Abbasi, N. A. (2013): Impact of exogenous application of salt and growth regulators on growth and yield of strawberry. - Pak. J. Bot. 45(4): 1179-1185.

[39] Rab, A., Haq, I. U. (2012): Foliar application of calcium chloride and borax influences plant growth, yield, and quality of tomato (Lycopersicon esculentum Mill.) fruit. - Turk. J. Agric. For. 36(6): 695-701.

[40] Rapisarda, P., Marisol, B., Pannuzo, P., Timpanaro, N. (2008): Effect of cold storage on vitamin $\mathrm{C}$, phenolics and antioxidant activity of five orange genotype (Citrus sinensis $\mathrm{L}$. osbeck). - Postharvest Biol. Technol. 49: 346-354.

[41] Ruck, J. A. (1969): Chemical Methods for Analysis of Fruits and Vegetables. - Res. Stat. Dept. Agri. Can., Summerland, pp. 27-30.

[42] Sams, C. E. (1999): Preharvest factors affecting postharvest texture. - Postharvest Biol. Technol. 15: 249-254.

[43] Sangha, B., Agehara, S. (2016): Optimization of growth-stage specific nitrogen fertilization improves strawberry growth and yield. - Proc. Fla. State Hort. Soc. 129: 137139.

[44] Santos, B. M., Chandler, C. K. (2009): Influence of nitrogen fertilization rates on the performance of strawberry cultivars. - Int. J. Fruit Sci. 9: 126-135.

[45] Sharma, R. R. (2002): Growing Strawberries. - International Book Distributing Co., Lucknow.

[46] Sun, F., Xia, X., Yin, W. (2009): The mutual regulations between ABA and calcium signal transduction pathways under abiotic stress. - Genomics and Applied Biology 28(2): 391-397.

[47] Taiz, L., Zeiger, E. (2006): Plant Physiology. $4^{\text {th }}$ Ed. - Sinauer Associates Inc., Sunderland, MA, pp. 207-208.

[48] White, P. J. (2000): Calcium channels in higher plants. - Biochimica et Biophysica Acta 1465: 171-189. 